REVIEW

\title{
SURGEON AND THE MACHINE - ROBOTS IN SURGERY
}

\author{
Baki Ekci ${ }^{1}$, Gokhan Agturk ${ }^{2}$ \\ ${ }^{1}$ Department of General Surgery, Halic University School of Medicine, Sutluce, Istanbul, Turkey \\ ${ }^{2}$ Department of Physiology, Halic University School of Medicine, Sutluce, Istanbul, Turkey \\ Corresponding author: Baki Ekci \\ E-mail:drbaki@yahoo.com
}

\begin{abstract}
The use of tools and machines in the field of medicine is very old, although the use of robots dates back to several decades. The purpose of using machinery and robots in the industry is to reduce production costs in the industry. Unlike machines, robots are energy-driven mechanical systems designed to perform learned operations and movements in a much safer faster and more economical way. In the medical sector, robots used outside operations are used to automate certain tasks. But the surgical robots are controlled by the surgeons and used to facilitate the surgeons' work. In other words, they do not move except for the surgeon's control and do not perform an automated procedure and they do not have artificial intelligence now. In this context, it is more appropriate to use the term robotic-assisted surgical equipment, robot-assisted minimally invasive surgery or roboticallyassisted surgical devices rather than using the word "robot". In short, robots used in surgeries are machines designed to perform more complex, thinner, more precise tasks. In this review, we will be evaluating the robot, the different medical assistants and robotic surgery, the da Vinci robot, and the differences between the open surgery, laparoscopic surgery, and robot-assisted surgery.
\end{abstract}

Keywords: robot, machinery, surgery

\section{Introduction}

In the recent years, there is a great improvement regarding the use of robotic technology in the field of surgery. Eventually, extensive research on surgical robots enabled this technique to move to operating rooms and furthermore, in certain surgeries, it has been used routinely. Since the surgical procedures are complex and require precision, the research in the field of robotic surgery has focused on the development of the robotic devices that are clinically sensitive and feasible. This leads the encouragement of the research in this field. However, the process of development requires a significant amount of financial resources and technical accumulation, which is the major drawback of the surgical robotics research.

\section{Materials and Methods}

An extensive literature search was conducted on PubMed, EMBASE, Scopus and Google Scholar with the keywords, robot, machinery, surgery, and da Vinci Surgical System. Inclusion criteria were studies from 1952 to present (2020), language in English, human studies, original research, case reports and reviews whereas exclusion criteria were studies published before 1952, non-English, animal studies, editorials, presentations and abstracts. 


\section{Results}

The machine (or mechanical device) is a mechanical structure that uses power and is controlled to perform an intended movement, whereas the tool is the mechanical device used to facilitate a task [1]. Robots are intelligent machines that can be programmed to perform complex tasks or combinations of tasks [2]. Machines are designed to help people in spending less labor and are things that make work easier or save time. In the medical field, especially in surgery, almost everything is done with the help of instruments. Needle holders and forceps are tools. In this step, we have to define "medical robot" as "robot" intended to be used as a medical device, because there is a difference between the robots used in the industrial sector and the medical robot $[3,4]$. According to the International Organization for Standardization, Medical robot is defined as "robot intended to be used as medical electrical equipment or medical electrical system" in IEC TR 60601-4-1. Medical electrical equipment/systems are terms roughly correspond to "active medical devices and systems using electric energy" $[3,5]$. The surgical machines called robots are controlled by the surgeon from the console, are able to perform movements that are difficult to be done by the human, increase the human's eyesight, reduce hand tremor and are designed to achieve difficult tasks in a narrow space $[3,6]$. As a working principle, a robot transmits images and information to the surgeon and moves within the patient in accordance with the commands it receives from the surgeon (Figure 1). For the first time in 1951, the Atomic Energy Commission (USA) designed an arm that holds hazardous materials [7]. Later on, machines that were described as robots doing repetitive work began to emerge. The first robot was named "robot surgeon", PUMA, and neurosurgical biopsies were performed with it $[8,9,10]$. Robots currently used in surgery have no automatic or intelligent self-movement and decision-making capabilities. The term robotic surgery (robot-assisted minimally invasive surgery or Robotic-Assisted Surgical Equipment) is used to mean the most common and typical application of robotic arms used actively in the field of surgery. The most famous surgical robot used for this work is the da Vinci Surgical System (Intuitive Surgery, Inc.,
Sunnyvale, CA, USA) [4]. The da Vinci ${ }^{\circledR}$ Robotic Surgical System (Intuitive Surgical Sunnyvale, CA) is the only device approved by the Food and Drugs Administration (FDA) [10]. The da Vinci robot master slave device has been developed by considering previous robots. It is the most commonly used surgical robot worldwide. The da Vinci abdominal was approved by the FDA in 2000 for abdominal surgery in humans $[10,11]$. Then, it was started to be used in surgery, gynecology, urology and obstetrics. With the development of new technologies, it has become more powerful, more practical and more usable over time. The da Vinci Surgical System has the following standard components: Surgeons console, Patient side cart, Endo-Wrist instruments and Vision System [12]. In generally, robotic surgery has advantages and disadvantages. It has very positive results when used in appropriate indication.

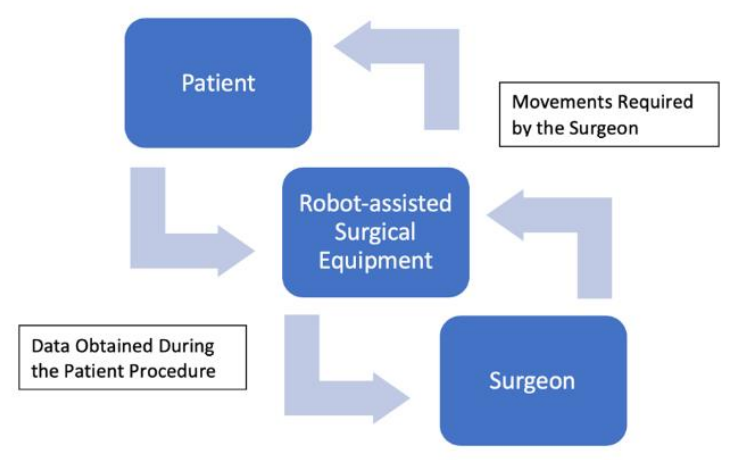

Figure 1 - Basic function of Robotic-Assisted Surgical Equipment

A surgical robot requires a large amount of capital. Other cost-increasing reasons include the use of a certain number of arms, their locking up and unavailability. High cost is also important in terms of accessibility and use. However, it has been stated that these cost rates may decrease with appropriate selection of the material to be used $[13,14]$. Nevertheless, the cost rate is very high compared to laparoscopic surgery [15]. Perhaps, the most important disadvantage is the lack of sense of touch by the surgeon. In addition, the time taken to prepare robotic structures before starting surgical procedures is longer than the conventional or laparoscopic surgery. This time may be shortened as the team experience increases $[13,14,16]$. In addition, because of the limited mobility of these machines, work areas are required in a single zone. Otherwise, surgeon 
needs to re-locate the robot, readjust it, maybe make a new incision. This is time consuming, laborious and exhausting. Now it is able to work in a number of fields for the new generation of robots, but it still does not fully provide human mobility and harmony. Medical robots are large machines, and they need larger operating rooms. So, this is the other disadvantage. Also; the presence of multiple connecting cables and components are potentially dangerous points in the use of this medical device. Another disadvantage is the need to change the arms such as scalpels, forceps, graspers, dissectors, cautery, scissors, retractors and suction irrigators, which are specially designed for different tasks during surgery [10].

The surgeon using the robot provides control from a place called the console. The presence of the body and arms on an ergonomic platform reduces the physical fatigue [17]. This, reduces the stress rate of the surgeon and supports a more balanced decision-making. In addition, the camera system is advanced; 3D image transfer, and magnification of the image help to see the details and give sense of depth. The most important deficiency of laparoscopic surgery is the perception of depth. The Endo-Wrist 'technology, which enables very delicate movements within the body, especially through a small incision, has enabled to perform sensitive works in narrow spaces. This feature is advantageous for intracorporal knotting in operations requiring precise dissection. It filters the vibrations that can be obtained especially in sensitive works and prevents the vibration in the last point. Another advantage is that the learning curve is shorter than laparoscopic surgery $[6,18,19,20,21]$. In addition, in the researches carried out with the help of this machine; decrease in hospital stay, decrease in postoperative pain and less need of analgesics, early return to daily activities of the patient, and good aesthetic results due to small incisions are important gains. In Table 1 and Table 2, you can see the comparison of open surgery, laparoscopic surgery and robotic surgery regarding patientand technique-related factors [1-23]. Compared to robotic surgery and laparoscopic surgery, although there are publications showing that there is no great difference between them, especially in the narrow areas such as pelvis and genitourinary surgery, the superiority of the functions in the preservation of surgery has been emphasized [21,22,23].

\begin{tabular}{lccc}
\hline & Open surgery & Laparoscopic surgery & Robot-assisted surgery \\
\hline Scarring rate & high & low & low \\
\hline Blood loss & high & low & low \\
\hline Post op pain & high & low & low \\
\hline Infection risk & high & low & low \\
\hline Complication rate & high & low & low \\
\hline Post op stay & high & low & low \\
\hline Recovery time & high & low & low \\
\hline
\end{tabular}

Table 1 - Patient-related factors associated with open surgery, laparoscopic surgery and robot-assisted surgery

\begin{tabular}{lccc}
\hline & Open surgery & Laparoscopic surgery & Robot-assisted surgery \\
\hline Compensate for hand tremor & no & no & yes \\
\hline Longer setup time & low & low & high \\
\hline Haptic feedback & yes & no & no \\
\hline Widely use area & high & high & low \\
\hline
\end{tabular}

Table 2 - Technique-related factors associated with open surgery, laparoscopic surgery and robotassisted surgery

The safety of medical robots used as medical devices or robotic surgery is also important. It is important to solve the safety issues, because it may be fatal to the patient [3]. The legal problems and responsibilities of robotic surgery, which is increasingly used widely, must be explained and 
regulated. It is obvious that this issue will be among the main research topics in the upcoming years, especially with the increase in the use of robot with or without artificial intelligence. We believe that there will be more research and publications on this subject.

In the future, considering the current situation, we think that with the development of new platforms and robotic surgery technology, the production of smaller and more portable robots or robotic arms might be possible, and even these robots will be sent down to the body and carry out their work there. In addition, the reduction and cheaper prices of robots are expected from this sector in the future. In the field of surgery, we believe that the word 'robot' will be the end point of working, deciding and applying with the artificial intelligence in order to gain full meaning.

\section{Conclusion}

As a result, it is really exciting to think about where to use robots in surgery. We believe that more ergonomic, flexible, high mobility devices will emerge. This is obviously not the last. As being excited about laparoscopic surgery, it is certain that the current situation in robotic surgery will progress further. This will be determined by expectations, difficulties, and the demands that will arise as you use them.

\section{References}

[1] https://wikidiff.com/machine/tool Machine vs Tool- What's the difference? (visited on Dec. 17, 2019)

[2] https://wikidiff.com/machine/robot Machine vs Robot- What's the difference? (visited on Dec. 17, 2019)

[3]K. Chinzei. Safety of Surgical Robots and IEC 80601-2-77: The First International Standard for Surgical Robots. Acta Polytechnica Hungarica, 16(8):171-84, 2019.

[4]Á. Takács, D. Nagy, I. Rudas, and T. Haidegger. Origins of Surgical Robotics: From Space to the Operating Room. Acta Polytechnica Hungarica, 13(1):13- 30, 2016.

[5] https://www.iso.org/standard/70755.html IEC/TR 60601-4-1:2017 Medical electrical equipment - Part 4-1: Guidance and interpretation - Medical electrical equipment and medical electrical systems employing a degree of autonomy. (visited on Dec. 17, 2019)

[6]R.N. Elek, and T. Haidegger. Robot-Assisted Minimally Invasive Surgical Skill AssessmentManual and Automated Platforms. Acta Polytechnica Hungarica, 16(8): 141-69, 2019.

[7]R.C. Goertz. Fundamentals of general-purpose remote manipulators. Nucleonics, 10(11):36-42, 1952.

[8]T.L. Ghezzi, and O.C. Corleta. 30 Years of Robotic Surgery. World J Surg, 40(10):2550-57, 2016.

[9]Y.S. Kwoh, J. Hou, E.A. Jonckheere, and S. Hayati. A robot with improved absolute positioning accuracy for CT guided stereotactic brain surgery. IEEE Trans Biomed Eng, 35(2):153-60, 1988.

[10] R. Valero, Y.H. Ko, S. Chauhan, O. Schatloff, A. Sivaraman, R.F. Coelho, F. Ortega, K.J. Palmer, R. Sanchez-Salas, H. Davila, X. Cathelineau, and V.R. Patel. Cirugía robótica: Historia e impacto en la enseñanza [Robotic surgery: history and teaching impact]. Actas Urol Esp, 35(9):540-5, 2011.

[11] US Food and Drug Administration (2000) 510 (k) clearances. https://www.accessdata.fda.gov/scripts/cdrh/cfdocs/c fpmn/pmn.cfm? ID=K002489\%20 (visited on Dec. 17, 2019)

[12] G.T. Sung, and I.S. Gill. Robotic laparoscopic surgery: a comparison of the Da Vinci and ZEUS systems. Urology, 58(6):893-8, 2001.

[13] Y. Lotan. Is robotic surgery cost-effective: no. Curr Opin Urol, 22(1): 66-9, 2012.

[14] J.C. Delto, G. Wayne, R. Yanes, A.M. Nieder, and A. Bhandari. Reducing robotic prostatectomy costs by minimizing instrumentation. J Endourol, 29(5):556-60, 2015.

[15] J.B. Mahida, J.N. Cooper, D. Herz, K.A. Diefenbach, K.J. Deans, P.C. Minneci, and D.J. McLeod. Utilization and costs associated with robotic surgery in children. J Surg Res, 199(1):169-76, 2015. [16] M.A. Talamini. Robotic surgery: Is it for you? Adv Surg, 36:1-13, 2002.

[17] J. Marescaux, J. Leroy, M. Gagner, F. Rubino, D. Mutter, M. Vix, S.E. Butner, and M.K. Smith. Transatlantic robot-assisted telesurgery. Nature, 413(6854):379-80, 2001.

[18] N. Taffinder, S.G. Smith, J. Huber, R.C. Russell, and A. Darzi. The effect of a second-generation 3D endoscope on the laparoscopic precision of novices and experienced surgeons. Surg Endosc, 13(11):1087-92, 1999.

[19] P. Yohannes, P. Rotariu, P. Pinto, A.D. Smith, and B.R. Lee. Comparison of robotic versus laparoscopic skills: is there a difference in the learning curve? Urology, 60(1):39-45, 2002, discussion 45 . 
[20] A. Blavier, Q. Gaudissart, G.B. Cadière, and A.S. Nyssen. Comparison of learning curves and skill transfer between classical and robotic laparoscopy according to the viewing conditions: implications for training. Am J Surg, 194(1):115-21, 2007.

[21] A.P. Aparna, R. Devika, and N. Kunhabdulla. Robotic interface controller for minimally invasive surgery. IJIRAE, 6(3): 163-6, 2019.

[22] F. Luca, M. Valvo, T.L. Ghezzi, M. Zuccaro, S. Cenciarelli, C. Trovato, A. Sonzogni, and R. Biffi.
Impact of robotic surgery on sexual and urinary functions after fully robotic nerve-sparing total mesorectal excision for rectal cancer. Ann Surg, 257(4):672-8, 2013.

[23] S.H. Lee, S. Lim, J.H. Kim, and K.Y. Lee. Robotic versus conventional laparoscopic surgery for rectal cancer: systematic review and meta-analysis. Ann Surg Treat Res, 89(4):190-201, 2015. 